\title{
Punções do estroma anterior no tratamento da ceratopatia bolhosa
}

\author{
Anterior stromal puncture in the treatment of bullous keratopathy
}

José Alvaro Pereira Gomes ${ }^{(1)}$

Daniel Keizo de Medeiros Haraguchi ${ }^{(2)}$

Delbis Uzcátegui Zambrano ${ }^{(3)}$

Luis Izquierdo Villavicencio ${ }^{(4)}$

Marcelo C. Cunha ${ }^{(5)}$
Denise de Freitas ${ }^{(6)}$

\section{RESUMO}

Objetivo: Avaliar o efeito terapêutico das punções do estroma anterior corneal em pacientes com ceratopatia bolhosa (CB).

Métodos: Vinte e cinco pacientes com $\mathrm{CB}$ sintomáticos, com baixa visão, com e sem indicação de transplante de córnea, foram avaliados antes, uma, 4 e 12 semanas após punções estromais anteriores realizadas com agulha \#25 à lâmpada de fenda. Em cada visita, os pacientes foram questionados sobre intensidade da dor, fotofobia, sensação de corpo estranho, além de serem submetidos a exame oftalmológico completo, estesiometria e paquimetria.

Resultados: As comparações realizadas entre os valores antes e após o procedimento referentes a dor $(p<0,001)$, fotofobia $(p=0,0198)$, sensação de corpo estranho $(\mathrm{CE})(\mathbf{p}<0,001)$, insônia $(p=0,0015)$ e estesiometria $(p=0,00654)$ apresentaram diferenças estatisticamente significantes quanto à diminuição desses sintomas e da sensibilidade corneal. A paquimetria média não apresentou diferença estatisticamente significante entre as avaliações antes e após as punções estromais $(p=0,873)$. Não foram observadas alterações importantes na vascularização corneal após o procedimento.

Conclusão: As punções do estroma anterior da córnea representam uma modalidade efetiva, simples e de baixo custo para o tratamento dos pacientes com CB sintomáticos.

Palavras-chave: Ceratopatia bolhosa; Edema de córnea; Tratamento.

\begin{tabular}{l} 
INTRODUÇão \\
\hline Ceratopatia bolhosa é uma doença da córnea causada pela descom- \\
pensação do endotélio secundária a trauma, glaucoma ou alterações congê- \\
nitas ${ }^{1}$. O resultado é a formação de edema estromal e bolhas epiteliais e \\
sub-epiteliais, que desencadeam diminuição da acuidade visual, dor, \\
fotofobia e epífora ${ }^{1,2}$. Os pacientes que aguardam ou não têm indicação de \\
transplante de córnea freqüentemente apresentam desconforto significati- \\
vo. Vários tratamentos são relatados na literatura, com resultados muitas \\
vezes frustrantes. Incluem-se o uso tópico de cloreto de sódio a $5 \%$, \\
antiinflamatórios não-hormonais, medicação anti-glaucomatosa, lentes de \\
contato terapêuticas, recobrimento conjuntival e cauterização ${ }^{1-3}$. \\
A punção do estroma anterior da córnea tem sido utilizada com sucesso no \\
tratamento de erosão recorrente, especialmente nos casos em que as lesões \\
estão localizadas fora do eixo visual ${ }^{4-7}$. O mecanismo de ação parece estar \\
relacionado com a penetração da membrana de Bowman pela agulha, que \\
induz a secreção de membrana basal e formação de novos complexos de \\
adesão entre epitélio e estroma subjacente ${ }^{4-8}$. Cormier et al. ${ }^{2}$ estudaram
\end{tabular}

Este trabalho foi realizado no Setor de Doenças Externas e Córnea do Depto. de Oftalmologia da Universidade Federal de São Paulo (UNIFESP).

(1) Mestre e pós-graduando nível doutorado, Setor de Córnea e Doenças Externas do Depto. de Oftalmologia da Universidade Federal de São Paulo(UNIFESP).

(2) Residente de $3^{\circ}$ ano do Depto. de Oftalmologia da Universidade Federal de São Paulo (UNIFESP).

(3) Ex-estágiaria do Setor de Córnea e Doenças Externas do Depto. de Oftalmologia da Universidade Federal de São Paulo (UNIFESP) no ano de 1997.

(4) Ex-residente do Depto. de Oftalmologia da Universidade Federal de São Paulo (UNIFESP).

(5) Doutor em oftalmologia e colaborador do Setor de Córnea e Doenças Externas do Depto. de Oftalmologia da Universidade Federal de São Paulo (UNIFESP).

(6) Doutora em oftalmologia e chefe do Setor de Córnea e Doenças Externas do Depto. de Oftalmologia da Universidade Federal de São Paulo (UNIFESP).

Endereço para correspondência: José Alvaro Pereira Gomes. Rua Sabará, 566 - Apto. 212. S. Paulo (SP) CEP 01239-010. Tel: (0xx11) 255-1603; Fax: (0xx11) 255-0223. e-mail: japgomes@uol.com.br 
clinicamente 27 pacientes portadores de ceratopatia bolhosa que foram submetidos a esse procedimento com bons resultados.

Em nosso meio, há vários pacientes portadores de ceratopatia bolhosa que apresentam intenso desconforto. Muitos deles têm indicação de ceratoplastia penetrante. Entretanto, a falta de córneas para transplante faz com que eles aguardem por longo tempo até serem submetidos a cirurgia. Há a necessidade de buscar alternativas simples, efetivas e de baixo custo para esses pacientes. O objetivo deste trabalho foi avaliar o efeito das punções no estroma corneal anterior nos portadores de ceratopatia bolhosa sintomáticos.

\section{PACIENTES E MÉTODOS}

Foi realizado estudo prospectivo de 25 pacientes seguidos no ambulatório de Córnea e Doenças Externas do Depto. de Oftalmologia da UNIFESP, portadores de ceratopatia bolhosa, que apresentavam sintomas apesar do uso de medicação tópica apropriada (agentes hiperosmóticos, lubrificantes e antinflamatórios), com baixa de acuidade visual e que aguardavam transplante de córnea ou não apresentavam indicação cirúrgica.

Os pacientes foram avaliados previamente ao tratamento (inicial ou 0), uma (1), 4 e 12 semanas após o tratamento. A avaliação constou de um formulário com os seguintes itens a serem preenchidos: intensidade da dor (0-3+), sensação de corpo estranho (0-3+), fotofobia (0-3+) e insônia relacionada à dor.

Além do questionário, foi realizada avaliação oftalmológica completa por dois dos autores (D.K.M.H., D.U.Z), que constou dos seguintes exames, repetidos a cada visita: biomicroscopia detalhada, na qual foram observadas a superfície da córnea com e sem fluoresceína, dobras de Descemet, vascularização superficial e profunda, fibrose sub-epitelial, presença de bolhas epiteliais e sub-epiteliais; desenho detalhado da evolução biomicroscópica do quadro; fotografias à lampâda de fenda; paquimetria ultra-sônica média (Humphrey Instruments Inc., San Leandro, EUA), calculada pela determinação da média da medida central e de 8 medidas periféricas equidistantes aproximadamente $3,5 \mathrm{~mm}$ do centro da córnea; sensibilidade corneal, calculada pela determinação da média de 3 medidas corneais centrais realizadas pelo estesiômetro de Cochet-Bonnet (Luneau, Paris, França); medida da pressão intra-ocular (tonômetro de aplanação).

\section{Procedimento}

As punções estromais foram realizadas pelo mesmo oftalmologista (J.A.P.G) da seguinte maneira: instilou-se uma gota de colírio de proparacaína $0,5 \%$ por 3 vezes no olho a ser submetido ao procedimento. Na lâmpada de fenda, foram realizadas aproximadamente 100 punções de estroma anterior em cada quadrante da córnea com uma agulha estéril \# 25 (Figuras 1 e 2). Após o procedimento, foi colocada uma lente de contato terapêutica, que foi retirada após 7 dias. No período pós-operatório, foi orientado aos pacientes instilar uma gota de

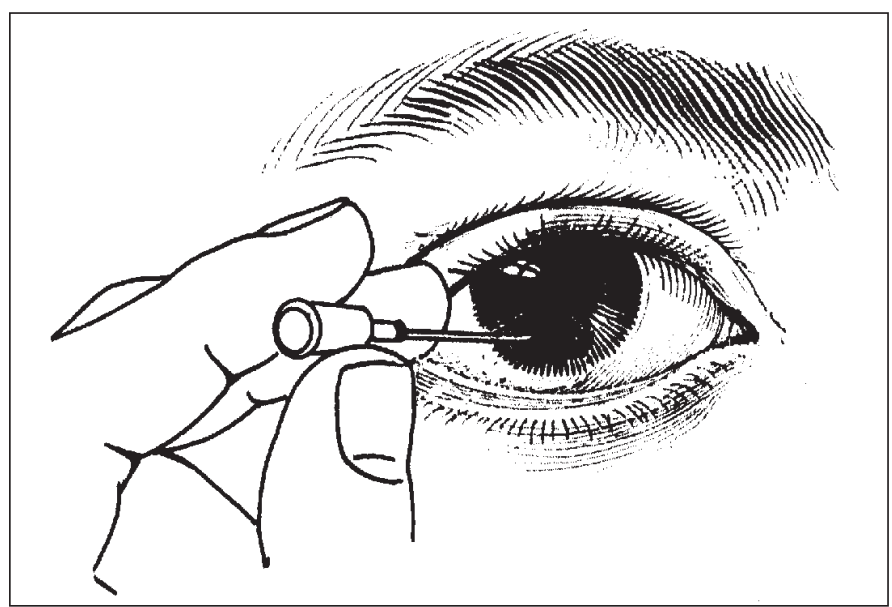

Fig. 1 - Desenho mostrando técnica da punção de estroma anterior da córnea.

Ofloxacina 3,0 $\mathrm{mg} / \mathrm{ml}$ quatro vezes por dia durante 7 dias e diclofenaco de sódio $1,0 \mathrm{mg} / \mathrm{ml}$ quatro vezes por dia durante 5 dias, além de manter medicação prévia. Nos pacientes que ainda apresentavam sintomas foi repetido o procedimento.

\section{Análise estatística}

Para avaliar uma possível concordância entre a observação da variável nas avaliações pré e pós-operatória final (12 semanas), foi utilizado o teste para mudanças de sinais de McNemar.

Para avaliar possíveis diferenças das variáveis contínuas nos vários períodos de avaliação ( $0,1,4$ e 12 semanas), foi usado o teste não-paramétrico para "K" amostras não-independentes de Friedman. Também na avaliação das variáveis subjetivas (discretas) dor, fotofobia e sensação de corpo estranho, foi aplicado o teste estatístico de Friedman, pois a finalidade era verificar se o comportamento dos indivíduos era o mesmo em cada período.

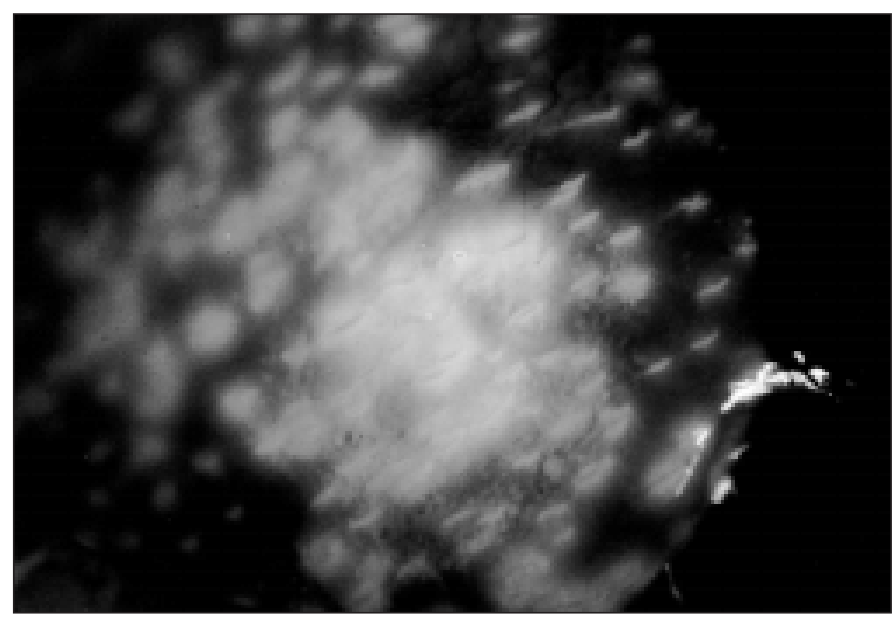

Fig. 2 - Aspecto biomicroscópico de uma córnea com ceratopatia bolhosa corada com fluoresceína $2 \%$ logo após as punções de estroma anterior (25x). 
O nível de rejeição fixado para a hipótese de nulidade foi sempre menor ou igual a $0,05(5 \%)$. Quando a análise estatística calculada apresentou significância, foi usado um asterisco (*) para caracterizá-la. Caso contrário, isto é, não-significante, foi usado "NS". As médias foram calculadas e apresentadas apenas a título de informação. Não foram calculados desvios-padrão, pois pressupondo-se que as variáveis não se comportam como curva de Gauss, não há sentido no cálculo do mesmo.

\section{RESULTADOS}

Foram analisados os resultados de 23 pacientes submetidos a punção estromal com 3 meses de seguimento. Dois pacientes foram excluídos do estudo por não terem completado o seguimento requerido. Todos os procedimentos foram completados com êxito sem nenhuma intercorrência. O tempo médio do procedimento foi de aproximadamente 4 minutos. Quatro pacientes $(17,4 \%)$ necessitaram ter as punções repetidas mais de uma vez, até referirem melhora significativa dos sintomas apresentados.

Dos 23 pacientes estudados, 13 eram do sexo feminino $(56,5 \%)$ e 10 do sexo masculino $(43,5 \%)$. A média da idade dos pacientes era de $60 \pm 17$ anos (variação entre 21 e 85 anos). Dez pacientes apresentavam como diagnóstico inicial ceratopatia bolhosa do pseudofácico (43,5\%), 8 como falência de transplante de córnea secundária a rejeição endotelial $(34,8 \%), 2$ como ceratopatia bolhosa do afácico $(8,7 \%), 2$ como ceratopatia bolhosa pós-trauma, $(8,7 \%)$ e 1 como distrofia hereditária congênita endotelial (4,3\%).

Um dos pacientes foi excluído da avaliação subjetiva por problemas neurológicos (deficiência de memória). Do total de 22 pacientes, $21(95,4 \%)$ apresentaram melhora da dor após a punção estromal. Um paciente $(4,5 \%)$ teve sua avaliação da dor após 3 meses inalterada em relação à inicial. Desses 22 pacientes, $11(50,0 \%)$ referiram ausência de dor 3 meses após o procedimento, o que representa melhora estatisticamente significante quando comparada com a de antes do procedimento ( $p=0,0026)$. A comparação entre as avaliações de dor realizadas inicialmente, 1,4 e 12 semanas após o procedimento apresentou-se estatisticamente significante $(\mathrm{p}<0,001)$. As comparações múltiplas revelaram que os pacientes sempre apresentaram melhora da dor em relação à avaliação inicial (tabela 1). Em relação a fotofobia, houve diferença estatisticamente significante na comparação entre as avaliações inicial, 1 , 4 e 12 semanas $(\mathrm{p}=0,0198)$. As comparações múltiplas mostraram diferença estatisticamente significante apenas entre as avaliações inicial e de 12 semanas (Tabela 1). Houve diminuição da sensação de corpo estranho em quase todos os pacientes.

Quanto a sensação de corpo estranho (CE), houve diferença estatisticamente significante na comparação entre as avaliações inicial, 1,4 e 12 semanas ( $p<0,001$ ). As comparações múltiplas mostraram que os pacientes sempre apresentaram melhora em relação à avaliação inicial (Tabela 1).

\begin{tabular}{|c|c|c|c|}
\hline \multirow[b]{2}{*}{ Comparações } & \multicolumn{2}{|r|}{ Variáveis } & \multirow[b]{2}{*}{ Sensação de CE } \\
\hline & Dor & Fotofobia & \\
\hline 0 vs. 1 vs. 4 vs. 12 & $X^{2}=36,64^{*}$ & $X^{2}=9,85^{*}$ & $X^{2}=26,47^{*}$ \\
\hline 0 vs. 1 & * & ns & * \\
\hline 0 vs. 4 & * & ns & * \\
\hline 0 vs. 12 & * & * & * \\
\hline 1 vs. 4 & ns & ns & ns \\
\hline 1 vs 12 & ns & ns & ns \\
\hline 4 vs. 12 & ns & ns & ns \\
\hline \multicolumn{4}{|c|}{$\begin{array}{l}0 \text { vs. } 1 \text { vs. } 4 \text { vs. } 12=\text { resultado do teste de Friedman; } 0 \text { vs. } 1,0 \text { vs. } 4,0 \text { vs. } 12 \text {, } \\
1 \text { vs. } 4,1 \text { vs. } 12,4 \text { vs. } 12=\text { resultado das comparações múltiplas; } C E=\text { corpo } \\
\text { estranho; ns=p>0,05; } *=p<0,05 .\end{array}$} \\
\hline
\end{tabular}

Os 10 pacientes $(45,4 \%)$ que apresentavam insônia em decorrência dos sintomas oculares antes do procedimento, relataram ausência da mesma após 3 meses do procedimento $(\mathrm{p}=0,0015)$.

A estesiometria apresentou diferença estatisticamente significante na comparação entre as avaliações inicial, 1, 4 e 12 semanas $(\mathrm{p}=0,00654)$. As comparações múltiplas mostraram diferença estatisticamente significante apenas entre as avaliações inicial e de 4 semanas, e inicial e de12 semanas (Tabelas 2 e 4).

\begin{tabular}{|ccccc|}
\hline \multicolumn{5}{|c}{$\begin{array}{c}\text { Tabela 2. Resultados da estesiometria antes, uma (1), } \mathbf{4} \text { e 12 } \\
\text { semanas após as punções estromais anteriores. }\end{array}$} \\
Pacientes & Antes & 1 semana & 4 semanas & 12 semanas \\
1 & 8,84 & 6,64 & 6,64 & 12,84 \\
2 & 12,84 & 8,84 & 12,84 & 17,68 \\
3 & 0,60 & 0,96 & 1,16 & 1,84 \\
4 & 3,20 & 3,20 & 4,60 & 4,60 \\
5 & 6,64 & 6,64 & 6,64 & 8,64 \\
6 & 1,40 & 2,40 & 17,68 & 17,68 \\
7 & 2,40 & 8,84 & 6,64 & 4,60 \\
8 & 2,40 & 4,60 & 0,96 & 2,40 \\
9 & 8,84 & 8,84 & 4,60 & 8,84 \\
10 & 12,84 & 17,68 & 17,68 & 17,68 \\
11 & 4,60 & 6,64 & 8,84 & 8,84 \\
12 & 6,64 & 6,64 & 6,64 & 3,20 \\
13 & 6,64 & 6,64 & 8,84 & 8,84 \\
14 & 4,60 & 4,60 & 12,84 & 6,64 \\
15 & 3,20 & 12,84 & 6,64 & 6,64 \\
16 & 3,20 & 6,64 & 8,84 & 8,84 \\
17 & 0,96 & 6,64 & 8,84 & 8,84 \\
18 & 17,68 & 17,68 & 17,68 & 17,68 \\
19 & 1,40 & 4,60 & 3,20 & 3,20 \\
20 & 1,40 & 12,84 & 12,84 & 8,84 \\
21 & 12,84 & 12,84 & 4,60 & 17,68 \\
22 & 8,84 & 6,64 & 8,84 & 8,84 \\
23 & 8,84 & 12,84 & 12,84 & 8,84 \\
Média & $\mathbf{6 , 1 4}$ & $\mathbf{8 , 1 2}$ & $\mathbf{8 , 7 4}$ & $\mathbf{9 , 2 9}$ \\
Mediana & $\mathbf{4 , 6 0}$ & $\mathbf{6 , 6 4}$ & $\mathbf{8 , 8 4}$ & $\mathbf{8 , 8 4}$ \\
\hline
\end{tabular}


A paquimetria média não apresentou diferença estatisticamente significante entre as avaliações inicial, 1, 4 e 12 semanas $(p=0,873)$ (Tabelas 3 e 4$)$.

Do total de 23 pacientes, apenas $4(17,4 \%)$ apresentaram piora em relação a neovascularização. Em 3 desses casos houve piora apenas da vascularização superficial e 1 caso apresentou piora da vascularização superficial e profunda. Em todos os pacientes foi observada diminuição significativa do número da bolhas epiteliais e sub-epiteliais e aumento da fibrose subepitelial com diminuição da transparência corneal.

\section{DISCUSSÃO}

Os pacientes portadores de ceratopatia bolhosa apresentam, muitas vezes, sintomas que interferem negativamente em sua qualidade de vida. Vários tratamentos são descritos na literatura para reduzir o desconforto e a dor apresentada por esses pacientes. A lente de contato terapêutica é uma alternativa simples, eficaz, porém associada ao aumento da vascularização e ao risco de infecção quando utilizada por tempo prolongado ${ }^{3}$. O uso de esteróides e agentes hiperosmóticos é preconizado por outros autores, porém com resultados limitados nos casos mais avançados da ceratopatia bolhosa ${ }^{1,2}$. A eletrocauterização da membrana de Bowman é um método efetivo para o tratamento dos casos avançados desta doença ${ }^{1,2}$. Entretanto, sua realização depende da presença do cautério e de ambiente cirúrgico adequado. Além disso,

\begin{tabular}{|c|c|c|c|c|}
\hline Pacientes & Antes & 1 semana & 4 semanas & 12 semanas \\
\hline 1 & 869 & 971 & 988 & 934 \\
\hline 2 & 762 & 797 & 863 & 885 \\
\hline 3 & 745 & 747 & 741 & 748 \\
\hline 4 & 694 & 695 & 733 & 733 \\
\hline 5 & 802 & 846 & 866 & 913 \\
\hline 6 & 801 & 750 & 746 & 808 \\
\hline 7 & 751 & 764 & 795 & 816 \\
\hline 8 & 837 & 802 & 812 & 676 \\
\hline 9 & 878 & 892 & 863 & 875 \\
\hline 10 & 1029 & 1021 & 1009 & 1025 \\
\hline 11 & 916 & 882 & 885 & 772 \\
\hline 12 & 757 & 763 & 765 & 691 \\
\hline 13 & 712 & 801 & 596 & 597 \\
\hline 14 & 972 & 1003 & 1003 & 959 \\
\hline 15 & 902 & 939 & 906 & 923 \\
\hline 16 & 799 & 790 & 797 & 813 \\
\hline 17 & 873 & 856 & 873 & 851 \\
\hline 18 & 912 & 802 & 846 & 874 \\
\hline 19 & 672 & 715 & 782 & 724 \\
\hline 20 & 870 & 868 & 835 & 865 \\
\hline 21 & 878 & 887 & 878 & 895 \\
\hline Média & 830,05 & 837,67 & 837,24 & 827,48 \\
\hline Mediana & 837 & 802 & 846 & 851 \\
\hline
\end{tabular}

\begin{tabular}{|c|c|c|}
\hline \multirow[b]{2}{*}{ Comparações } & \multicolumn{2}{|c|}{ Variáveis } \\
\hline & Estesiometria & Paquimetria média \\
\hline 0 vs. 1 vs. 4 vs. 12 & $X^{2}=12,26^{*}$ & $\mathrm{X}^{2}=0,96(\mathrm{~ns})$ \\
\hline 0 vs. 1 & ns & - \\
\hline 0 vs. 4 & * & - \\
\hline 0 vs. 12 & $\star$ & - \\
\hline 1 vs. 4 & ns & - \\
\hline 1 vs. 12 & ns & - \\
\hline 4 vs. 12 & ns & - \\
\hline \multicolumn{3}{|c|}{$\begin{array}{l}0 \text { vs. } 1 \text { vs. } 4 \text { vs. } 12=\text { resultado do teste de Friedman; } 0 \text { vs. } 1,0 \text { vs. } 4,0 \text { vs. } 12 \text {, } \\
1 \text { vs. } 4,1 \text { vs. } 12,4 \text { vs. } 12 \text { = resultado das comparações múltiplas; ns=p>0,05; } \\
\begin{array}{l}*=0<0,05 .\end{array}\end{array}$} \\
\hline
\end{tabular}

pode induzir modificações acentuadas na curvatura da córnea, vascularização e até necrose estromal, o que pode prejudicar a realização de transplante de córnea no futuro ${ }^{1,2}$.

A técnica da punção estromal anterior da córnea representa uma alternativa simples, de baixo custo, podendo ser realizada ambulatorialmente com o auxílio da lâmpada de fenda ${ }^{47}$. No presente trabalho, pudemos comprovar a praticidade do procedimento. Todos os pacientes toleraram bem a realização das quatrocentas punções, que foram concluídas em menos de 5 minutos. A escolha da agulha \# 25 para a realização das punções deveu-se ao trabalho de Katsev et al., ${ }^{9}$ que relata ser esta a agulha que melhor penetra os $2 / 3$ anteriores da córnea com segurança e menor trauma do tecido vizinho. Pode-se inclusive dobrar a ponta da agulha para dar maior segurança ao procedimento.

Cormier et al. ${ }^{2}$ relataram bons resultados deste procedimento no tratamento da ceratopatia bolhosa. Os resultados do presente trabalho confirmam os achados desses autores no que se refere a diminuição dos sintomas, principalmente em relação a dor. Praticamente todos pacientes por nós avaliados tiveram diminuição de dor após a realização das punções estromais e, em aproximadamente 50\% dos casos, houve ausência de dor após o procedimento. Foi também observada diminuição significativa da fotofobia e sensação de corpo estranho na maioria dos pacientes.

É importante salientar a dificuldade em aferir variáveis subjetivas como dor, fotofobia, sensação de corpo estranho e insônia. Neste trabalho, realizamos essa avaliação de maneira idêntica durante todo o seguimento dos pacientes, que tiveram a oportunidade de comparar os sintomas antes e após o tratamento. A pergunta feita aos pacientes em relação a intensidade dos sintomas pareceu ser adequada e facilmente compreensível. Outro método de avaliação subjetiva por nós testado inclui a escala analógica, ${ }^{2}$ que não foi utilizada neste trabalho pela dificuldade encontrada por esse grupo de pacientes em preenchê-la.

Como Cormier et al. ${ }^{2}$, também observamos aumento significante nos valores da estesiometria após a realização das 
punções estromais, o que significa que houve diminuição da sensibilidade corneal após o procedimento. Este achado foi evidenciado somente após 4 semanas do procedimento. A paquimetria média não apresentou diferença significante antes e depois do tratamento, o que contrasta com os achados de Cormier et al. ${ }^{2}$, que encontrou aumento da espessura corneal após 3 meses das punções estromais. Talvez diferenças na técnica da punção estromal ou na realização da paquimetria possam explicar essa diferença ${ }^{10}$.

Dúvidas ainda persistem com relação ao mecanismo de ação das punções no estroma e ao porquê da melhora dos sintomas relatados pelos pacientes ${ }^{2,8}$. Sabe-se que o rompimento das bolhas está relacionado com as crises de dor referidas pelos pacientes, possivelmente pela exposição das terminações nervosas da córnea ${ }^{1}$. A teoria que afirma que as punções introduziriam projeções epiteliais ancorantes no estroma anterior não foi totalmente comprovada ${ }^{2,8}$. Mais recentemente, Hsu et al. ${ }^{8}$ demonstraram, através de técnicas imunohistoquímicas, aumento da expressão de proteínas da matriz extra-celular importantes na adesão das células epiteliais basais (fibronectina, laminina e colágeno tipo IV) nos locais das punções estromais realizadas em olhos com ceratopatia bolhosa. A secreção desses componentes da membrana basal aumentaria a adesão epitelial no estroma subjacente que, associada a formação de fibrose sub-epitelial, criaria uma barreira à penetração de líquido no espaço subepitelial e, conseqüentemente, diminuição da formação das bolhas sub-epiteliais ${ }^{2}$.

Notamos que, nas áreas em que existiam bolhas, estas diminuíram e até desapareceram após as punções. Após alguns dias, notou-se a formação de fibrose sub-epitelial, o que teoricamente ajudaria na formação da barreira a penetração de líquido e nova formação de bolhas. O aumento da vascularização foi discreto e principalmente superficial, o que representa uma vantagem em relação a outros procedimentos mais agressivos ${ }^{1}$.

Em resumo, o tratamento da ceratopatia bolhosa com punções estromais anteriores diminui os sintomas dos pacientes, em especial a dor por eles referida. Este procedimento é de fácil realização, eficiente e de baixo custo, e deve ser considerado para o tratamento desses pacientes. Mais tempo de seguimento, no entanto, é necessário para melhor avaliar seu efeito terapêutico na ceratopatia bolhosa.

\section{SUMMARY}

Purpose: To evaluate the effect of anterior stromal puncture to treat bullous keratopathy patients $(B K)$ who were symptomatic. Methods: Twenty-five patients with BK and decreased vision, awaiting or not penetrating keratoplasty, were evaluated before, 1, 4 and 12 weeks after anterior stromal punctures performed with a \# 25 needle. The patients were asked about pain intensity, foreign body sensation and photophobia. Biomicroscopy, esthesiometry and pachymetry results were evaluated.

Results: The comparisons between results before and after the anterior stromal puncture related to pain $(p<0.001)$, photophobia $(p=0.0198)$, foreign-body sensation $(p<0.001)$, insomnia $(p=0.0015)$ and esthesiometry $(p=0.00654)$ showed significant statistical differences regarding decreasing symptoms and corneal sensitivity. The mean pachymetry did not show a statistical difference between results before and after the procedure $(p=0.873)$. We did not find important differences in terms of corneal neovascularization.

Conclusion: Anterior stromal puncture is an effective, simple and low cost alternative to treat symptomatic patients with $B K$.

Keywords: Bullous keratopathy; Corneal edema; Treatment.

\section{REFERÊNCIAS BIBLIOGRÁFICAS}

1. Aquavella JV. Corneal edema. In: Leibowitz HM, Ed. Corneal disorders: Clinical diagnoses and management. Philadelphia, PA: WB Saunders Co; 1984:164-82.

2. Cormier G, Brunette I, Boisjoly HM, LeFrançois M, Shi ZH, Guertin M-C. Anterior stromal punctures for bullous keratopathy. Arch Ophthalmol 1996;114:654-8.

3. Plotnnik RD, Mannis MJ, Schwab IR. Therapeutic contact lens. Int Ophthalmol Clin 1991;31:35-52.

4. Rubinfeld RS, Laibson PR, Cohen EC, Arentsen JJ, Eagle Jr., RC. Anterior stromal punctures for recurrent erosion: further experience and new instrument. Ophthalmic Surg 1990;21:318-26.

5. Mc Lean EN, Mc Rae SM, Rich LF. Recurrent erosion treatment by anterior stromal puncture. Ophthalmology 1986;93:784-8.

6. Kenyon KR. Recurrent corneal erosion: pathogenesis and therapy. Int Ophthalmol Clin 1979;9:169-95.

7. Galbavy EJ, Mobilia EF, Kenyon KR. Recurrent corneal erosion. Int Ophthalmol Clin 1984;24:107-31.

8. Hsu JKW, Rubinfeld RS, Barry P, Jester JV. Anterior stromal punctures: imunohistochemical studies in human corneas. Arch Ophthalmol 1993;111: 1057-63.

9. Katsev DA, Kincaid MC, Fouraker BD, Dresner MS, Schanzlin DI. Recurrent corneal erosion: pathology of corneal puncture. Cornea 1991;10:418-23.

10. Tanaka HM, Mori ES, Maia NS, Juliano Y, Campos M. Espessura corneana na alta miopia. Arq Bras Oftalmol. 1996;59:295-8.

\section{Novidades na Internet!! !}

Agora no site CBO você tem disponível todas as informações na íntegra dos

Arquivos Brasileiros de Oftalmologia

http: / / www.cbo.com.br/abo 